\title{
Intra-abdominal pathology in the dissecting room: a study of incidence and its value in teaching
}

\author{
Dan Magrill, Reza Mirnezami, Harold Ellis \\ Department of Anatomy, Guy's Campus, Guy's King's and St Thomas' School of Biomedical Sciences, King's College, London, United Kingdom
}

\begin{abstract}
Having systematically studied the abdominal cavities of all cadavers dissected by the first year of medical students at our institution, this paper presents the results. Thirty six cadavers were dissected, and the students took an active role in the data collection of visceral pathology and anatomical variations encountered. It was shown how, in the vast majority of cadavers $(89 \%)$, intra-abdominal pathology was seen by the medical students. The most common were gallstones (28\%), hepatic metastases (25\%), colonic diverticula (25\%) and adhesions (25\%). Two particularly interesting cases are presented in detail, along with photographs and histological slides. It is also noted that this pathology can be used to educate the students about common disease states that they will encounter in clinical practice.
\end{abstract}

Key words: anatomical sciences; medical education; anatomy teaching; pathology; histopathology; undergraduate medical education

Anatomy 2008; 2: 22-27, (c) 2008 TSACA

\section{Introduction}

The practice of cadaveric dissection has long been considered to be the principal method to instruct medical students in human anatomy. In the setting of the dissection room, medical students develop a sense of respect and of compassion, and are provided with the opportunity to explore the human body in detail and under the supervision of qualified anatomists. ${ }^{1}$ They acquire knowledge of the fundamentals of normal human anatomy and also develop a basic appreciation of biological variations and pathological conditions.

The Department of Anatomy at King's College London is one of the departments in the United Kingdom where teaching by dissection still exists. Currently cadaveric teaching, once considered pivotal in the training of the medical undergraduate, is facing a cri- sis with growing controversy concerning its continuing role in the teaching of gross anatomy. Some suggest that a combination of computer assisted learning, supplemented with surface anatomy and medical imaging can provide medical students with adequate knowledge of gross anatomy in the absence of cadaver-based education. $^{2}$

In the light of the changes currently proposed by some authorities, this study aims to illustrate that anatomical dissection not only plays a pivotal role in the instruction of normal gross human anatomy, but that it also provides students with a valuable introduction to commonly encountered surgical pathologies. ${ }^{3}$ This will be illustrated in the context of pathology encountered in the dissection room with respect to the abdominal cavity, and specifically the gastrointestinal viscera. 


\section{Materials and Methods}

The medical students at Guy's, King's and St Thomas' School of Medicine performed abdominal, thoracic and pelvic dissection of 36 cadavers in the Anatomy Department between August 2005 and May 2006.

For each cadaver, a pro-forma was completed by the Anatomy Demonstrator and the group of 10-15 students assigned to each body. The demographics for each body was completed by the authors.

Following external examination for any obvious abnormalities or indications of pathology, and dissection of the anterior abdominal wall, the students proceeded to examine macroscopically the alimentary canal and its adnexae.

This study assessed primarily visceral intra-abdominal pathology. Vascular, musculoskeletal and genitourinary abnormalities detected during dissection of the abdomen were not specifically studied but were encouraged to be noted on the pro-forma under "Other" .

\section{Results}

There were 18 female and 18 male bodies in this series, and the average age at death was $81 \mathrm{yrs}$ old. "Consequences of Cancer" was the most common cause of death (53\%) (as given on the death certificate), followed by "Heart Failure" (22\%), "Bronchopneumonia" (17\%), "Cerebro-vascular Accident" (6\%) and "Respiratory Failure" (3\%).

In only four $(11 \%)$ of the bodies no intra-abdominal pathology recorded, and the only anatomical abnormality discovered in one of these was an undescended testis. A total of 70 pathologies were found in the 36 bodies, which are listed in Table $\mathbf{1 .}$

Three patients had had a colectomy (8\%), two had a bowel stoma $(6 \%)$ and two had had a cholecystectomy $(6 \%)$. Stents were found in the oesophagus, common bile duct, pancreatic duct and ureter. Other operative procedures noted included a gastro-jejunal bypass, and axillofemoral arterial bypass graft, a nephrectomy, a hysterectomy and an abdominal aortic aneurysm graft.
Table 1

Incidence of pathologies

\begin{tabular}{lc}
\hline Gallstones & $10(28 \%$ of bodies) \\
Hepatic metastases & $9(25 \%)$ \\
Adhesions & $9(25 \%)$ \\
Diverticula & $9(25 \%)$ \\
Hepatomegaly & $3(8 \%)$ \\
Cirrhosis & $3(8 \%)$ \\
Colorectal cancer & $3(8 \%)$ \\
Renal cyst & $2(6 \%)$ \\
Abdominal aortic aneurysm & $2(6 \%)$ \\
Uterine fibroids & $2(6 \%)$ \\
Inguinal hernia & $2(6 \%)$ \\
Renal cancer & $2(6 \%)$ \\
Fatty change to liver & $2(6 \%)$ \\
Gastric cancer & $2(6 \%)$ \\
Peptic ulcer disease & $2(6 \%)$ \\
Colonic stricture & $2(6 \%)$ \\
Splenomegaly & $1(3 \%)$ \\
Ovarian tumour & $1(3 \%)$ \\
Umbilical hernia & $1(3 \%)$ \\
Renal atrophy & $1(3 \%)$ \\
Oesophageal cancer & $1(3 \%)$ \\
Pancreatic cancer & $1(3 \%)$ \\
\hline
\end{tabular}

In two cases, the macroscopic appearances were so remarkable and of such interest to the student and demonstrators that histological examination was performed to ascertain the precise nature of the lesions.

\section{Cadaver 1 - Gross hepatomegaly}

One of the cadavers was an 81 year old man who had died of metastatic rectal cancer. The striking finding was the size of his liver, which took up the entire visible abdominal cavity (Figure 1). It measured $30 \mathrm{~cm}$ (length) x $25 \mathrm{~cm}$ (width) x $17 \mathrm{~cm}$ (depth) and weighed over $11 \mathrm{~kg}$.

A section taken (Figure 2) showed that almost the entire tissue had been replaced by metastatic tumour with only a small amount of liver tissue remaining at the edge of the organ. This had compressed all of the other abdominal organs, and there was evidence of several operations including a hemicolectomy and stoma formation. The primary tumour was also dissected from the anterior wall of the rectum. 
Figure 1. Liver in situ.

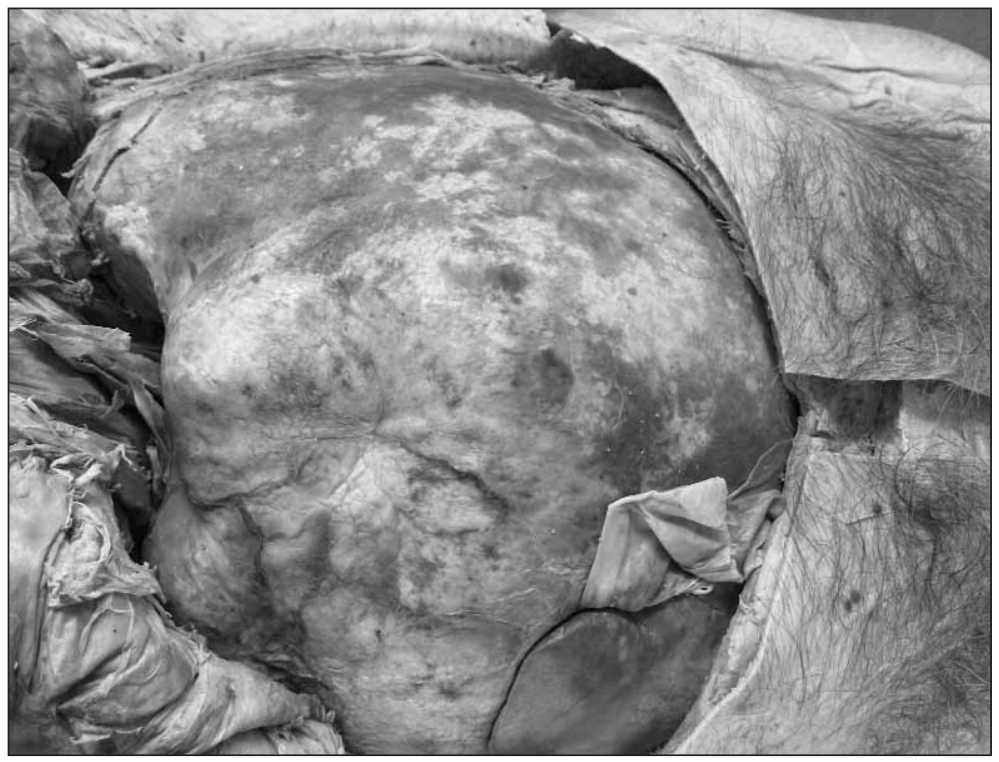

Figure 2. Cut surface of liver.

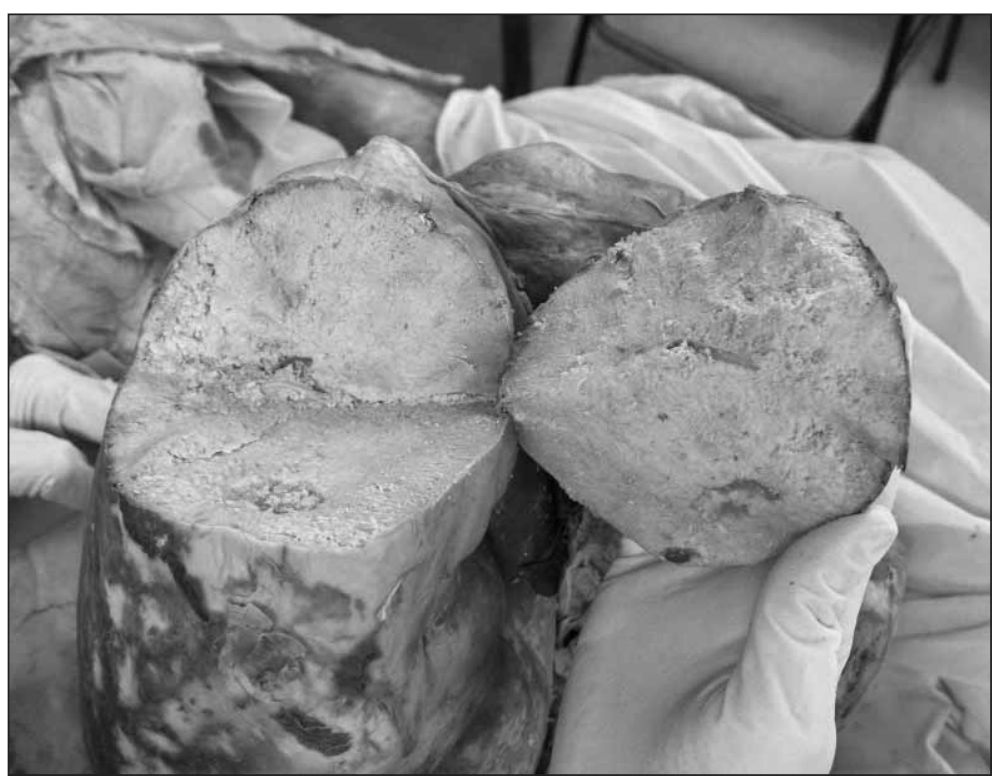

\section{Cadaver 2 - Peritoneal deposits}

The second cadaver of note was that of an 81 year old female who died of advanced breast cancer, and had undergone a left mastectomy. When the abdominal cavity was opened, it was found that the abdominal wall, the peritoneum and many of the organs were covered in deposits (Figures 3 and 4).

Small biopsies were fixed in formalin, embedded and processed into paraffin blocks. Sections (4 micrometers thick) were cut from each block and stained with
Heamatoxylin and Eosin (H\&E) according to standard protocols.

Each of the H\&E stained sections were examined microscopically, showing nodular deposits of tumour, consisting of large cells with moderately pleomorphic nuclei and a moderate amount of eosinophilic cytoplasm. At the periphery of some of the nodular deposits tumour cells were arranged in rows, reminiscent of lobular carcinoma of the breast (Figure 5, magnified $\mathrm{x} 400)$. 
The sections submitted from the uterus revealed infiltration through the myometrium by individual tumour cells, with a similar morphology to those cells forming the nodules in the omentum and mesentery.

Paraffin-embedded tissues were sectioned at 4 micrometers and mounted onto Electrostatic Adhexive slides (Surgipath, Peterborough, UK). Tissue sections were deparaffinised in xylene and hydrated to water through graded alcohol concentrations. For antigen retrieval they were pressure cooked for 2 minutes using $0.1 \mathrm{~mol} / \mathrm{l}$ citrate buffer ( $\mathrm{pH} 6.0)$ to unmask the antigens.
Slides were immersed in 1:60 hydrogen peroxide for 20 minutes to block endogenous peroxidase activity. For immunohistochemical detection of ER and PR, slides were incubated in a dark chamber for 30 minutes at room temperature with 1:50 dilution of mouse ER monoclonal antibody (Neomarkers, Fremont, Canada) or 1:75 dilution of mouse PR monoclonal antibody (Novocastra, Newcastle-Upon-Tyne, UK) both in antibody diluent (Dako, Cambridge, UK). For negative controls, antibody diluent was used without the primary antibody.
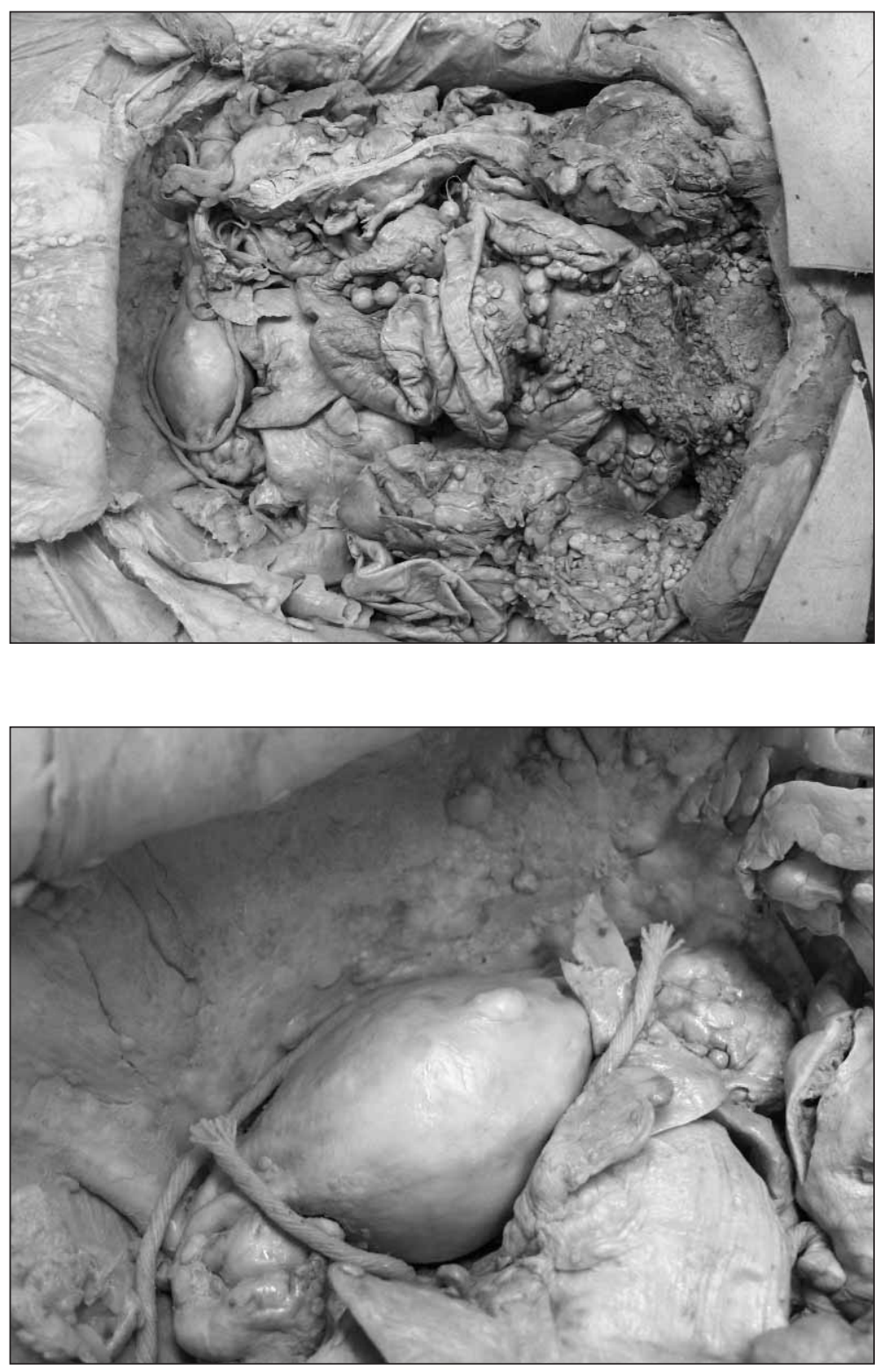

Figure 3. Overview of peritoneal deposits.

Figure 4. Deposits on uterus. 
Bound antibody was visualized using a polymer-based secondary labeling system (Envision, Dako, Cambridge, UK) for 30 minutes, according to the manufacturers instructions. The reaction product was uncovered by $\mathrm{DAB}$ and substrate buffer (Dako, Cambridge, UK) for 10 minutes. Counterstaining was carried out with Harris' Haematoxylin for 20 seconds.

This immunohistochemistry showed that the tumour cells were negative for ER and PR, although the receptor status of the original tumour was unknown. The morphological appearances were in keeping with metastatic breast carcinoma.

\section{Discussion}

This study into the pathology found in the cadavers that are studied by first year medical students gives insight into how the dissection room is not only an excellent place to learn anatomy, but also how it is an opportunity for students to learn about pathology.

In nearly $90 \%$ of the groups of students, some pathology would have been demonstrated and used as a point of discussion. By the very nature of common pathologies such as gallstones, diverticula and adhesions being found so often, many of the students will have seen first hand the disease states that they will encounter in their clinical attachments. The way that anatomy is taught in this particular dissecting room, with 12 groups working at one time, means that the demonstrators can take groups of students round to see the pathologies and anatomical variations found in other cadavers.

The fact that so many of the cadavers used had known pathology (such as cancer) did surprise the authors. In addition, concerns have been raised that so much pathology might remove opportunities for the students to learn normal anatomy from the cadavers. This is minimized, to an extent, by the groups of students being able to move between cadavers.

As the great majority of anatomy demonstrators at this particular University are surgical trainees, ${ }^{4}$ the 20 operations that the cadavers in our study had received could be explained to the students. This could form the basis of discussions of patient management the treatment of disease. The dissecting room provides an environment where an integrated and problem solving approach to learning can thrive.

The case of hepatomegaly discussed highlights the extremes of pathology that may be witnessed in a dissecting room. Initially drawn to this cadaver by the size of the liver, the demonstrators were then able to teach on the causes and consequences of hepatomegaly while the students had the experience reinforced by visual and tactile pathways only available in a dissecting room. ${ }^{1}$

Figure 5. Tumour cells arranged in rows (x400 mag).

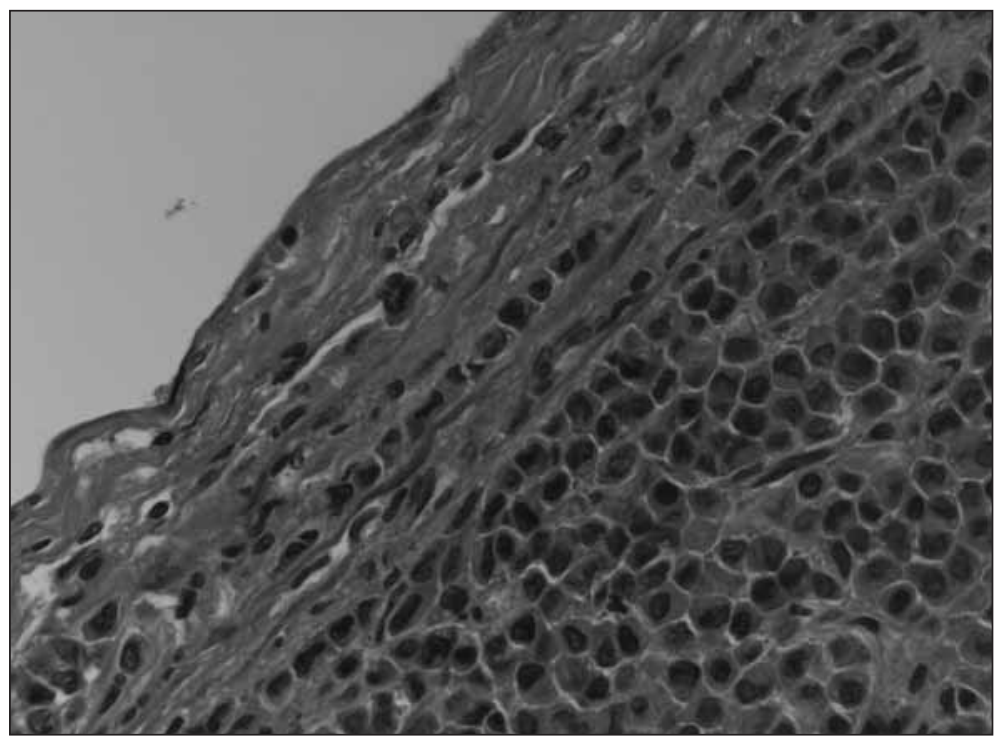


The case of peritoneal metastases had similar advantages. By involving the students in the follow up of this cadaver they were interested and excited by the links between anatomy, histology and pathology that it provided. Not only were the demonstrators now able to talk about the spread of cancer, but the students were also given an insight into the diagnostic processes that will be encountered in their clinical experience.

Having involved the students in the data collection of this study, they also gained their first exposure to scientific research, and many continued to ask the authors about the results of the study and the process of research.

As mentioned, those who feel anatomy teaching using methods other that cadavers may see the amount of pathology found in this study as distracting from the normal anatomy that is being taught. For this reason the authors feel that a good case is made here for a special study module to be created into Surgical Anatomy and Pathology. It would bee helpful to involve pathologists in this.

In conclusion, the authors believe that this study highlights the usefulness of cadaveric teaching in a stu- dent's education not only of anatomy, but also of pathology. They are able to get first-hand experience of disease states and receive focused and informative teaching from surgical trainees.

\section{Acknowledgement}

The authors would like to acknowledge the kind help of Dr. Horsfield and Frances Biggins of the Histopathology Department at St Thomas' Hospital, London, who kindly donated a lot of time and effort to help with the histopathology.

\section{References}

1. Granger NA. Dissection laboratory is vital to medical gross anatomy education. Anat Rec (New Anat) 2004; 281 B: 6-8.

2. Mc Lachlan JC. New path for teaching anatomy: living anatomy and medical imaging vs. dissection. Anat Rec (New Anat) 2004; 281 B: $4-5$.

3. Ellis H. Teaching in the dissection room. Clin Anat 2001; 14: 149151.

4. Gossage JA, Horner B, Halsey T, Ellis H. The importance of anatomy demonstrating for the surgical trainee. A Bulletin for Surgeons in Training. Surgery (Scalpel) 2002; 20: 1-2.

Correspondence to: Dan Magrill, MD 9 Westmead, London SW15 5BH, UK Phone: +44 (0) 7801801 789; Fax: +44 (0) 2087886003 e-mail: danmagrill@hotmail.com

Conflict of interest statement: No conflicts declared. 